\title{
Almost disjoint families and property (a)
}

by

Paul J. Szept y cki (Athens, Ohio) and

Jerry E. V a ughan (Greensboro, N.C.)

\begin{abstract}
We consider the question: when does a $\Psi$-space satisfy property (a)? We show that if $|\mathcal{A}|<\mathfrak{p}$ then the $\Psi$-space $\Psi(\mathcal{A})$ satisfies property (a), but in some Cohen models the negation of $\mathrm{CH}$ holds and every uncountable $\Psi$-space fails to satisfy property (a). We also show that in a model of Fleissner and Miller there exists a $\Psi$-space of cardinality $\mathfrak{p}$ which has property (a). We extend a theorem of Matveev relating the existence of certain closed discrete subsets with the failure of property (a).
\end{abstract}

1. Introduction. A space $X$ has property (a) [13] provided for every open cover $\mathcal{U}$ and dense set $D$ of $X$ there exists a closed discrete (in $X$ ) $F \subset D$ such that $\operatorname{st}(F, \mathcal{U})=X$. Property (a) was introduced by Matveev in order to explore the absoluteness condition in the definition of absolutely countable compactness [11]. Some results on property (a) can be found in [9], [13], and [14].

In the first part of this paper we consider the question: Under what conditions does the space $\Psi(\mathcal{A})$ satisfy property (a)? Recall that for an almost disjoint family $\mathcal{A}$ of infinite subsets of $\omega, \Psi(\mathcal{A})$ denotes the associated topological space whose underlying set consists of the set of natural numbers $\omega$, and one point $x_{A}$ for every $A \in \mathcal{A}$. The points in $\omega$ are declared to be isolated, and basic neighborhoods of a point $x_{A}$ are of the form $\left\{x_{A}\right\} \cup(A \backslash n)$ for all $n \in \omega$ (see $[6,5 \mathrm{I}]$, or $[3,3.6 . \mathrm{I}]$ ). Evidently, if $\mathcal{A}$ is countable, then $\Psi(\mathcal{A})$ has property (a); so we are only interested in uncountable almost disjoint families. The statement " $\Psi(\mathcal{A})$ has property (a)" translates into the following set-theoretic statement about $\mathcal{A}$ :

$(*) \quad(\forall f: \mathcal{A} \rightarrow \omega)(\exists P \subset \omega)(\forall A \in \mathcal{A})(0<|P \cap(A \backslash f(A))|<\omega)$.

We generalize in $\S 5$ the following theorem which can be applied to $\Psi(\mathcal{A})$.

1991 Mathematics Subject Classification: 54A25, 54A35, 03E50, 04-01, 03E05, 04A20.

Key words and phrases: property (a), density, extent, almost disjoint families, $\Psi$-space, $\mathrm{CH}, \mathrm{GCH}$, Martin's Axiom, $\mathfrak{p}=\mathfrak{c}$, Cohen forcing, Q-set, weakly inaccessible cardinal. 
Theorem 1 (M. Matveev [13]). If $X$ is separable, and contains a closed discrete subset of cardinality $\mathfrak{c}$, then $X$ does not have property (a).

COROllary 1. If $|\mathcal{A}|=\mathfrak{c}$, then $\Psi(\mathcal{A})$ does not satisfy property (a).

It is known, and easy to see, that if $\mathcal{A}$ is maximal (i.e., $\Psi(\mathcal{A})$ is pseudocompact), then $\Psi(\mathcal{A})$ does not satisfy property (a) [14]. We recall that there are models of Hechler [7] in which the cardinality of the continuum $\mathfrak{c}$ is arbitrarily large and there exist maximal almost disjoint families of every uncountable cardinality less than or equal to $\mathfrak{c}$. Part of our motivation for this paper was to find what other conditions on $\mathcal{A}$ imply that $\Psi(\mathcal{A})$ has (or does not have) property (a). While this problem is primarily motivated by topological concerns its analysis led us to some new questions about almost disjoint families which may be of independent interest.

Our results yield the following one concerning models of set theory:

Theorem 2. (1) $[\mathfrak{p}=\mathfrak{c}] \Rightarrow \Psi(\mathcal{A})$ satisfies property (a) if and only if $|\mathcal{A}|<\mathfrak{c}$.

(2) Adding any number of Cohen reals to a model of $\mathrm{CH}$ results in a model where $\Psi(\mathcal{A})$ satisfies property (a) if and only if $\mathcal{A}$ is countable.

(3) There is a model with an almost disjoint family $\mathcal{A}$ of size $\mathfrak{p}$ where $\Psi(\mathcal{A})$ satisfies property (a).

It is well known that $\mathfrak{p}=\omega_{1}$ in the Cohen model used in part (2) of Theorem 2; so in the models used in both parts (1) and (2) of Theorem 2, every $\Psi$-space of size $\mathfrak{p}$ fails to satisfy property (a). This motivates part (3) of Theorem 2. The proof of Theorem 2 is given in Sections 2, 3, and 4 .

To generalize Theorem 1, we introduce in $\S 5$ two new cardinal invariants related to density and extent, and we study these further in $\S 7$. In $\S 6$ we consider the effect of property (a) on the inequality $e(x)<2^{d(X)}$. Some open questions are given in $\S 8$. We use certain small cardinals throughout the paper; see [2] and [5].

2. Martin's Axiom and $\Psi(\mathcal{A})$. Recall that a family $\mathcal{P}$ of subsets of the natural numbers has the strong finite intersection property provided every finite intersection of members of $\mathcal{P}$ is infinite. An infinite $S \subset \omega$ is a pseudointersection for $\mathcal{P}$ provided $S \backslash P$ is finite for all $P \in \mathcal{P}$ (see [2]).

Definition 1. $\mathfrak{p}=\min \left\{|\mathcal{P}|: \mathcal{P} \subset[\omega]^{\omega}\right.$ has the strong finite intersection property, but has no pseudointersection\}.

We use two basic facts about the cardinal $\mathfrak{p}$ :

(1) (Bell's theorem [1], [5, 14C]) if $\kappa<\mathfrak{p}$, then "MA for $\sigma$-centered posets of cardinality $\kappa$ " holds, and

(2) Martin's Axiom implies that $\mathfrak{p}=\mathfrak{c}[5,11 \mathrm{D}]$. 
We say that an almost disjoint family $\mathcal{A}$ is soft if there exists $P \subseteq \omega$ such that for all $A \in \mathcal{A}, 0<|P \cap A|<\omega$. It is obvious that any family $\mathcal{A}$ that satisfies $(*)$ is soft, and that there exist soft families of every cardinality $\leq \mathfrak{c}$ (thus a soft family $\mathcal{A}$ of cardinality $\mathfrak{c}$ does not satisfy $(*)$ ). We use the following observation: If $\kappa$ is a cardinal such that every almost disjoint family $\mathcal{A}$ with $|\mathcal{A}|<\kappa$ is soft, then every almost disjoint family $\mathcal{A}$ with $|\mathcal{A}|<\kappa$ satisfies $(*)$; hence the corresponding $\Psi(\mathcal{A})$ satisfies property (a).

Theorem 3. If $|\mathcal{A}|<\mathfrak{p}$ then $\Psi(\mathcal{A})$ satisfies property (a).

Pr o o f. It suffices to prove that any almost disjoint family $\mathcal{A}$ with $|\mathcal{A}|<$ $\mathfrak{p}$ is soft. This can can be deduced directly from Fremlin's "portmanteau theorem" [5, 21A]. We sketch a proof that is a slight variation on the proof that "MA $\Rightarrow \mathfrak{a}=\mathfrak{c}$ " $[10,2.16]$. Let $\mathcal{A}$ with $|\mathcal{A}|<\mathfrak{p}$ be given. According to $[10,2.7]$ "the almost disjoint sets partial order" $\mathbb{P}_{\mathcal{A}}$ is defined to be

$$
\left\{(s, F): s \in[\omega]^{<\omega}, F \in[\mathcal{A}]^{<\omega}\right\},
$$

where $\left(s^{\prime}, F^{\prime}\right) \leq(s, F)$ if and only if

$$
s \subset s^{\prime}, \quad F \subset F^{\prime} \quad \text { and } \quad(\forall A \in F)\left(A \cap s^{\prime} \subset s\right) .
$$

We use the following subset:

$$
\mathbb{Q}_{\mathcal{A}}=\left\{(s, F) \in \mathbb{P}_{\mathcal{A}}: \text { for every } A \in F, s \cap A \neq \emptyset\right\} .
$$

Since $\left(\mathbb{P}_{\mathcal{A}}, \leq\right)$ is a $\sigma$-centered poset, so is $\left(\mathbb{Q}_{\mathcal{A}}, \leq\right)$. Moreover, for each $A \in \mathcal{A}$,

$$
D_{A}=\left\{(s, F) \in \mathbb{Q}_{\mathcal{A}}: A \in F\right\}
$$

is dense in $\mathbb{Q}_{\mathcal{A}}$. MA says that there is a filter $G \subset \mathbb{Q}_{\mathcal{A}}$ such that $G \cap D_{A} \neq \emptyset$ for all $A \in \mathcal{A}$. Then $P=\bigcup\{s:(\exists F)((s, F) \in G)\}$ is the desired set.

\section{Cohen forcing and $\Psi(\mathcal{A})$}

THEOREM 4. In the Cohen model, $\Psi(\mathcal{A})$ does not have property (a) whenever $\mathcal{A}$ is an uncountable almost disjoint family.

Proof. We force with $\operatorname{Fn}(\kappa, 2)$ over a model $\mathcal{M}$ of $\mathrm{CH}$. If $\kappa \leq \omega_{1}$ the result follows from Corollary 1; so we assume that $\kappa>\omega_{1}$. Fix an $\mathcal{M}$-generic set $G \subseteq \operatorname{Fn}(\kappa, 2)$. We claim that in $\mathcal{M}[G]$ the conclusion of the theorem holds. To show this, fix an uncountable almost disjoint family $\mathcal{A} \in \mathcal{M}[G]$. It is easy to check that if $\Psi\left(\mathcal{A}_{0}\right)$ does not have property (a) for some subset $\mathcal{A}_{0} \subseteq \mathcal{A}$ then $\Psi(\mathcal{A})$ will also fail to have property (a). Therefore we may assume that $\mathcal{A}$ is of size $\omega_{1}$. Therefore there is an $I \subseteq \kappa$ of size $\omega_{1}$ such that $\mathcal{A} \in \mathcal{M}[G \cap \operatorname{Fn}(I, 2)]$. Note that $\mathrm{CH}$ holds in this intermediate model, and that $\mathcal{M}[G]$ is obtained by forcing with $\operatorname{Fn}(\kappa \backslash I, 2)$ over $\mathcal{M}[G \cap \operatorname{Fn}(I, 2)]$. Therefore we may also assume that $\mathcal{A}$ is in the ground model $\mathcal{M}$. Working with the negation of $(*)$, we now construct an open cover of $X=\Psi(\mathcal{A})$, also 
in the ground model, witnessing that $X$ does not have property (a) in the extension. This open cover will be of the form

$$
\mathcal{U}_{f}=\{\{a\} \cup(a \backslash f(a)): a \in \mathcal{A}\} \cup\{\{n\}: n \in \omega\}
$$

where $f: \mathcal{A} \rightarrow \omega$. We need the following lemma.

Lemma 1. Assume $C H$. Let $\mathcal{A}$ be an almost disjoint family of size $\omega_{1}$. There is a function $f: \mathcal{A} \rightarrow \omega$ such that for each $\operatorname{Fn}(\omega, 2)$-name $\tau$ for a subset of $\omega$, if

(a) $\mathbf{1} \Vdash(\forall a \in \check{\mathcal{A}})(|\tau \cap a|<\omega)$

then

(b) $\mathbf{1} \Vdash(\exists a \in \check{\mathcal{A}})(\tau \cap(a \backslash \check{f}(a))=\emptyset)$.

Proof. Enumerate $\mathcal{A}$ as $\left\{a_{\alpha, n}: \alpha<\omega_{1}, n<\omega\right\}$, and by CH, enumerate all $\operatorname{Fn}(\omega, 2)$-nice names for subsets of $\omega$ as $\left\{\tau_{\alpha}: \alpha<\omega_{1}\right\}$. Fix $\alpha<\omega_{1}$. If there is a $p \in \operatorname{Fn}(\omega, 2)$ and an $a \in \mathcal{A}$ such that

$$
p \Vdash|\check{a} \cap \tau|=\omega
$$

then let $f\left(a_{\alpha, n}\right)=0$ for each $n \in \omega$. So assume that

(c) $\mathbf{1} \Vdash(\forall a \in \check{\mathcal{A}})\left(\left|\tau_{\alpha} \cap a\right|<\omega\right)$.

It is well known that $\operatorname{Fn}(\omega, 2)$ does not add any function in ${ }^{\omega} \omega$ dominating all ground model functions in ${ }^{\omega} \omega$. In the language of forcing extensions this means that if $G$ is $\operatorname{Fn}(\omega, 2)$-generic over $\mathcal{M}$ then for each $s \in{ }^{\omega} \omega \cap \mathcal{M}[G]$ there is an $f \in{ }^{\omega} \omega \cap \mathcal{M}$ such that $\{n: f(n)>s(n)\}$ is infinite (see [10], Exercise VII.G7). Therefore

$$
\begin{aligned}
E=\{ & p \in \operatorname{Fn}(\omega, 2): \\
& \left.\left(\exists f \in{ }^{\omega} \omega\right)\left(p \Vdash “ \check{f}(n)>\max \left(\tau_{\alpha} \cap \check{a}_{\alpha, n}\right) \text { for infinitely many } n "\right)\right\}
\end{aligned}
$$

is a dense subset of $\operatorname{Fn}(\omega, 2)$. For each $p \in E$ fix a corresponding $f_{p} \in{ }^{\omega} \omega$. Since $E$ is countable, we may choose $g_{\alpha} \in{ }^{\omega} \omega$ such that for each $p \in E$, $g_{\alpha}(n)>f_{p}(n)$ for all but finitely many $n \in \omega$. Then

(d) $\mathbf{1} \Vdash\left\{n \in \omega: \check{g}_{\alpha}(n)>\max \left(\tau_{\alpha} \cap \check{a}_{\alpha, n}\right)\right\}$ is infinite.

(We will only need the fact that this set is forced to be nonempty). Now define $f: \mathcal{A} \rightarrow \omega$ by $f\left(a_{\alpha, n}\right)=g_{\alpha}(n)$ for each $\alpha \in \omega_{1}$ and each $n \in \omega$. If

(e) $\mathbf{1} \Vdash(\forall a \in \check{\mathcal{A}})(|\tau \cap \check{a}|<\omega)$,

then there is an $\alpha$ such that

(f) $\mathbf{1} \Vdash \tau=\tau_{\alpha}$.

Therefore, by (d),

$$
\mathbf{1} \Vdash(\exists n \in \omega)\left(\tau_{\alpha} \cap \check{a}_{\alpha, n} \subseteq \check{g}_{\alpha}(n)\right) .
$$


But this clearly means that

$$
\mathbf{1} \Vdash(\exists a \in \check{\mathcal{A}})\left(\tau_{\alpha} \cap(a \backslash \check{f}(a))=\emptyset\right) .
$$

This completes the proof of Lemma 1.

To complete the proof of Theorem 4, fix $G \subseteq \operatorname{Fn}(\kappa, 2)$ generic over $\mathcal{M}$, and fix $f$ given by the lemma. We claim that in $\mathcal{M}[G], X$ does not have property (a). By way of contradiction assume otherwise. Then, since $\omega$ is dense in $X$, there is an $F \subseteq \omega$ closed discrete such that $\operatorname{st}\left(F, \mathcal{U}_{f}\right)=X$. By (*), $F \cap a$ is finite for each $a \in \mathcal{A}$ and $F \cap(a \backslash f(a)) \neq \emptyset$ for each $a \in \mathcal{A}$. Since $F$ is countable, there is a countable $I \subseteq \kappa$ such that $F \in \mathcal{M}[G \cap \operatorname{Fn}(I, 2)]$. But as $\operatorname{Fn}(I, 2)$ is isomorphic to $\operatorname{Fn}(\omega, 2)$ and $G \cap \operatorname{Fn}(I, 2)$ is $\operatorname{Fn}(I, 2)$-generic over $\mathcal{M}$, there is an $\operatorname{Fn}(\omega, 2)$-name $\tau$ such that

$$
\mathbf{1} \Vdash(\forall a \in \check{\mathcal{A}})(|\tau \cap a|<\omega \text { and } \tau \cap(a \backslash \check{f}(a)) \neq \emptyset) .
$$

This contradicts Lemma 1.

4. A $\Psi$-space of size $\mathfrak{p}$ with property (a). We prove that it is consistent that there is an almost disjoint family $A$ of size $\mathfrak{p}$ with property (a). Since the existence of a $Q$-set implies the existence of a normal $\Psi$-space of size $\omega_{1}$, the existence of $A$ follows from the next two theorems. Recall that $\mathfrak{d}$ denotes the smallest cardinality of a dominating family of functions from $\omega$ to $\omega$ with respect to the mod finite order (e.g., see [2]).

TheOREM 5. Suppose that $A \subseteq[\omega]^{\omega}$ is an almost disjoint family such that $|A|<\mathfrak{d}$ and such that $\Psi(A)$ is normal. Then $\Psi(A)$ has property (a).

Theorem 6 (Fleissner-Miller). If ZFC is consistent then so is ZFC+ (there is a $Q$-set $)+\left(\mathfrak{d}=2^{\omega}=\omega_{2}\right)+\left(\mathfrak{p}=\omega_{1}\right)$.

Proof of Theorem 5. We need some notation. For a finite sequence $s \in \omega^{n}$ and $k \in \omega, s \frown k \in \omega^{n+1}$ is the sequence extending $s$ whose $(n+1)$ st element is $k$. For sequences $s, t \in \omega^{n}$ we write $s \leq t$ if $s(k) \leq t(k)$ for each $k<n$.

Fix $A$ as in the hypothesis of the theorem. It suffices to prove that any such $A$ is soft. For each $s \in \omega^{<\omega}$ define $A_{s} \subseteq A$ and open sets $U_{s}, V_{s} \subseteq \Psi(A)$ as follows. $A_{\emptyset}=\emptyset, U_{\emptyset}=\emptyset$ and $V_{\emptyset}=\Psi(A)$. For each $n \in \omega$ let $A_{\langle n\rangle}=\{a \in$ $A: a \cap n \neq \emptyset\}$. Using normality of $\Psi(A)$ fix disjoint open sets $U_{\langle n\rangle} \supseteq A_{\langle n\rangle}$ and $V_{\langle n\rangle} \supseteq A \backslash A_{\langle n\rangle}$. In addition we choose these open sets so that

(a) $n<m$ implies $U_{\langle n\rangle} \subseteq U_{\langle m\rangle}$ and $V_{\langle m\rangle} \subseteq V_{\langle n\rangle}$.

Fix $n>1$ and suppose that $A_{s}, U_{s}$ and $V_{s}$ have been defined for each $s \in \omega^{n}$ so that

(b) $A_{s} \subseteq A, U_{s}$ and $V_{s}$ are open in $\Psi(A)$ such that $U_{s} \cap V_{s}=\emptyset$ for each $s \in \omega \leq n$.

(c) $U_{s} \supseteq \bigcup_{i \leq n} A_{s \mid i}$ and $V_{s} \supseteq A \backslash \bigcup_{i \leq n} A_{s \mid i}$ are disjoint open sets. 
(d) $U_{s \mid i} \subseteq U_{s \mid j}$ and $V_{s \mid i} \supseteq V_{s \mid j}$ for each $i<j \leq n$ and for each $s \in \omega^{n}$.

Fix $s \in \omega^{n+1}$. Let

$$
A_{s}=\left\{a \in A \backslash \bigcup_{i \leq n} A_{s \mid i}: a \cap(s(n) \backslash s(n-1)) \cap V_{s \mid n} \neq \emptyset\right\} .
$$

Using normality, fix a clopen $U \subseteq \Psi(A)$ such that $U \cap A=A_{s}, U \cap U_{s \mid n}=\emptyset$ and $U \subseteq V_{s \mid n}$. Let $U_{s}=U_{s \mid n} \cup U$ and let $V_{s} \subseteq V_{s \mid n}$ be an open set such that $A \backslash \bigcup_{i \leq n+1} A_{s \mid i} \subseteq V_{s}$ and $U_{s} \cap V_{s}=\emptyset$. This completes the construction of the family $\left\{A_{s}, U_{s}, V_{s}: s \in \omega^{<\omega}\right\}$. Notice that the sets $A_{s}$ satisfy the following properties.

(e) For each $s \in \omega^{n}$ and for each $k>j \geq s(n-1), A_{s \frown j} \subseteq A_{s \frown k}$.

(f) For each $s \in \omega^{n}, A=\bigcup_{i \leq n} A_{s \mid i} \cup \bigcup_{k>s(n-1)} A_{s \frown k}$.

For each $a \in A$ define $f_{a}: \omega \rightarrow \omega$ as follows. Let $f_{a}(0)$ be the minimum $k$ such that $a \in A_{\langle k\rangle}$. For $n>1$, having defined $f_{a} \mid n$ let $f_{a}(n)$ be the minimum $k$ such that for each $s \in \omega^{n}$, if $s \leq f_{a} \mid n$ and $a \notin \bigcup_{i \leq n} A_{s \mid i}$, then $a \in A_{s \frown k}$. Clause (f) and the fact that the set of such $s$ is finite guarantee that $f_{a}(n)$ is well defined.

Using $|A|<\mathfrak{d}$, fix an increasing $f \in \omega^{\omega}$ such that for each $a \in A$ there is an $n \in \omega$ such that $f(n) \geq f_{a}(n)$.

Lemma 2. $A=\bigcup_{n \in \omega} A_{f \mid n}$.

Proof. Fix $a \in A$. Fix $n$ minimal so that $f(n) \geq f_{a}(n)$. Suppose that $a \notin \bigcup_{i \leq n} A_{f \mid i}$. Since $f\left|n \leq f_{a}\right| n, a \in A_{(f \mid n) \frown f_{a}(n)}$ by the definition of $f_{a}(n)$. By clause (e), $a \in A_{(f \mid n) \frown k}$ for each $k \geq f_{a}(n)$ so $a \in A_{f \mid n+1}$.

Now we define $X$ that will witness that $A$ is soft. Let $S_{0}=f(0)$. For each $n>0$ let $S_{n}=(f(n) \backslash f(n-1)) \cap V_{f \mid n}$ and let $X=\bigcup_{n \in \omega} S_{n}$. Fix $a \in A_{f \mid n+1}$. By the definition of $A_{f \mid n+1}, a \cap S_{n} \neq \emptyset$ and by (b) and (d), $a \in U_{f \mid n+1} \subseteq$ $U_{f \mid m}$ for all $m>n$. By our construction we also have $U_{f \mid n+1} \cap V_{f \mid m}=\emptyset$ for each $m>n$. Therefore, $a \cap \bigcup_{m>n} S_{m} \subseteq a \backslash U_{f \mid n+1}$. But since $U_{f \mid n+1}$ is an open set containing $a, a \backslash U_{f \mid n+1}$ is finite and therefore $a \cap \bigcup_{m>n} S_{m}$ is finite. Therefore $0<|a \cap X|<\omega$ as required.

Proof of Theorem 6. One of the models of [4] satisfies the conclusion of the theorem. Fleissner and Miller construct a model where there is a Q-set concentrated on a countable set. Therefore $\mathfrak{b}=\omega_{1}$ in this model (see Theorem 10.2 in [2]) and hence $\mathfrak{p}=\omega_{1}$. The forcing used to obtain the model is an $\omega_{2}$ length finite support iteration of CCC partial orders. Therefore any set of reals of size $\omega_{1}$ appears at some initial stage $\alpha<\omega_{2}$. And since it is a finite support iteration, $V^{P_{\alpha+\omega}}$ contains a Cohen real over $V^{P_{\alpha}}$ which is therefore not dominated by any real in $V^{P_{\alpha}}$. So no set of reals of size $\omega_{1}$ is dominating. 
5. Density discreteness and cofinality. Recall the definition of density of a space $X$ :

$$
d(X)=\min \{|D|: D \text { is a dense subset of } X\}+\omega .
$$

Matveev's proof of Theorem 1 yields the following more general statement.

Theorem 7 (Matveev). If $X$ has a closed discrete set $F$ with $|F| \geq 2^{d(X)}$, then $X$ does not satisfy property (a).

In order to extend this result we introduce two new cardinal invariants.

Notation. For a dense set $D \subset X$, let $\mathcal{F}_{D}$ denote the set of all closed discrete (in $X$ ) subsets of $D$.

Definition 2. The density discreteness number of a space $X$ is

$$
d d(X)=\min \left\{\left|\mathcal{F}_{D}\right|: D \text { is dense in } X\right\}+\omega .
$$
$2^{d(X)}$

Clearly, $d d(X) \leq 2^{d(X)}$, and if $X$ is a $T_{1}$-space, then $d(X) \leq d d(X) \leq$

Let $\mathcal{F}$ be a family of sets. We recall that $\mathcal{C} \subset \mathcal{F}$ is said to be cofinal in $\mathcal{F}$ provided for every $F \in \mathcal{F}$ there exists $C \in \mathcal{C}$ such that $F \subset C$.

Definition 3. The density discreteness cofinality of a space $X$ is the following number:

$$
d d c(X)=\min \left\{|\mathcal{C}|: D \text { is dense in } X \text { and } \mathcal{C} \text { is cofinal in } \mathcal{F}_{D}\right\}+\omega .
$$

Obviously, $d d c(X) \leq d d(X)$, but $d d c(X)<d(X)$ is possible. Indeed, consider the following

EXAmple 1. There exists a metrizable space $X$ such that $d d c(X)<$ $d(X)<d d(X)$.

Proof. Let $X=\left(\omega_{1} \times \omega\right) \cup\{\infty\}$ where the points of $\omega_{1} \times \omega$ are isolated, and the sets $B(\infty, n)=\{\infty\} \cup\left\{(\alpha, i): i>n, \alpha<\omega_{1}\right\}$ for $n<\omega$ form a base at $\infty$. Now $D=\omega_{1} \times \omega$ is dense in $X$ and $\mathcal{C}=\left\{\omega_{1} \times n: n<\omega\right\}$ is cofinal in $\mathcal{F}_{D}$ since every set in $X$ that intersects infinitely many levels $\left(\omega_{1} \times\{n\}\right)$ has $\infty$ as a limit point; so $d d c(X)=\omega<\omega_{1}=d(X)<2^{\omega_{1}}=d d(X)$.

TheOREM 8. If $X$ has a closed discrete subset $F$ with $|F| \geq d d c(X)$, and the interior of $F$ is empty, then $X$ does not satisfy property (a).

Proof. The proof uses the main idea in Matveev's proof of Theorem 1. Let $D$ be dense and $\mathcal{C}_{D}$ cofinal in $\mathcal{F}_{D}$ such that $d d c(X)=\left|\mathcal{C}_{D}\right|$. Since the interior of $F$ is empty, $D \backslash F$ is dense in $X, \mathcal{F}_{D \backslash F} \subset \mathcal{F}_{D}$,

$$
\mathcal{C}=\left\{H \backslash F: H \in \mathcal{C}_{D}\right\}
$$

is cofinal in $\mathcal{F}_{D \backslash F}, C \cap F=\emptyset$ for all $C \in \mathcal{C}$, and $|\mathcal{C}| \leq\left|\mathcal{C}_{D}\right|=d d c(X)$; so $|\mathcal{C}|=d d c(X)$. 
Let $d d c(X)=\kappa$, list $\mathcal{C}$ as $\left\{H_{\alpha}: \alpha<\kappa\right\}$, and list $\kappa$ points of $F$ as $G=$ $\left\{x_{\alpha}: \alpha<\kappa\right\}$. For each $\alpha<\kappa$, get an open set $U_{\alpha}$ such that $U_{\alpha} \cap G=\left\{x_{\alpha}\right\}$, and $U_{\alpha} \cap H_{\alpha}=\emptyset$. Then the open cover $\mathcal{U}=\left\{U_{\alpha}: \alpha<\kappa\right\} \cup\{X \backslash G\}$ and the dense set $D \backslash F$ demonstrate that $X$ does not satisfy property (a): If $P \subset D \backslash F$ is closed discrete in $X$, then for some $\alpha<\kappa$,

$$
\operatorname{st}(P, \mathcal{U}) \subset \operatorname{st}\left(C_{\alpha}, \mathcal{U}\right) \subset X \backslash\left\{x_{\alpha}\right\} .
$$

This completes the proof.

The hypothesis " $F$ has nonempty interior" in Theorem 8 cannot be deleted. The space $X$ in Example 1 provides a counterexample since any space with exactly one nonisolated point obviously satisfies property (a).

Corollary 2. If $X$ has a closed discrete subset $F$ with $|F| \geq d d(X)$, then $X$ does not satisfy property (a).

Proof. Put $G=\{x \in F:\{x\}$ is open in $X\}$. It suffices to show that $|G|<|F|$ because we can then apply Theorem 8 to $F \backslash G$. Suppose $|G|=|F|$. Since $G$ is a set of isolated points, $G \subset D$ for every dense set $D$. Thus $\left|\mathcal{F}_{D}\right| \geq$ $2^{|G|}=2^{|F|}$; so we have $2^{|F|} \leq d d(X)$. But this leads to the contradiction

$$
d d(X) \leq|F|<2^{|F|} \leq d d(X) .
$$

Since $d d(X) \leq 2^{d(X)}$, Corollary 2 shows immediately that Matveev's Theorem 7 is a corollary to Theorem 8 . Also, we note that Corollary 2 can be used to show that $\Psi(\mathcal{A})$ does not have property (a) whenever $|\mathcal{A}|=\mathfrak{c}$ or $\mathcal{A}$ is maximal.

6. Extent. We recall the definitions of extent of a space $X$ :

$$
e(X)=\sup \{|F|: F \text { is a closed discrete subset of } X\}+\omega .
$$

In general the two numbers $e(X)$ and $d d c(X)$ are not related. For the space $X$ in Example 1, we have $d d c(X)<e(X)$, and for the space $X=$ $L\left(\omega_{1}\right)$, the one-point Lindelöfization of the discrete space of size $\omega_{1}$, we have $e(X)<d d c(X)$. Both of these spaces satisfy property (a).

Corollary 3. If $X$ has property $(\mathrm{a})$, then $e(X) \leq d d(X)$. In particular, $e(X) \leq 2^{d(X)}$.

Proof. By contradiction, if $e(X)>d d(X)$, then there exists a closed discrete set $F$ with $|F| \geq d d(X)$; so by Corollary $2, X$ does not satisfy property (a), which is a contradiction.

It is known that for any regular space $X, e(X) \leq w(X) \leq 2^{d(X)}[8$, $3.3(\mathrm{~b})]$. Thus property (a) and regularity both imply $e(X) \leq 2^{d(X)}$. On the other hand, property (a) implies $e(X) \leq d d(X)$, and regularity does not (if $X=\Psi(\mathcal{A})$ where $\mathcal{A}$ is maximal, then $\left.d d(X)=\omega<e(X) \geq \omega_{1}\right)$. Of course, regularity does not yield a strict inequality in either case (if $X=\Psi(\mathcal{A})$ 
where $\mathcal{A}$ is not maximal and $|\mathcal{A}|=\mathfrak{c}$, then $\left.e(X)=d d(X)=2^{d(X)}=\mathfrak{c}\right)$. Likewise, property (a) does not imply a strict inequality in the first case (if $X$ is a compact separable space, then $e(X)=d d(X)=\omega)$.

We are left with the following question: Does property (a) imply $e(X)<$ $2^{d(X)}$ ? We show that an affirmative answer to this question is consistent with and (assuming a certain kind of inaccessible cardinal) independent of the usual axioms of ZFC. Let $\mathcal{S}$ stand for the following statement:

$$
\mathcal{S} \text { : "If } X \text { has property (a), then } e(X)<2^{d(X)} \text { ". }
$$

We will use the set-theoretic assumption " $2^{\kappa}$ is a successor cardinal for each cardinal $\kappa "$. This assumption is implied by $\mathrm{GCH}$, and is consistent with $\mathrm{MA}+\neg \mathrm{CH}$. We also use the assumption " $\mathfrak{p}=\mathfrak{c}$ and $\mathfrak{c}$ is weakly inaccessible" (concerning the consistency of this assumption, see [10, VII, Cor. 6.5]).

THEOREM 9. (i) If $2^{\kappa}$ is a successor cardinal for each cardinal $\kappa$ then $\mathcal{S}$.

(ii) If $\mathfrak{p}=\mathfrak{c}$ and $\mathfrak{c}$ is weakly inaccessible then $\neg \mathcal{S}$.

Pr o of of (i). We prove $\mathcal{S}$ by contrapositive; so we assume that $e(X) \geq$ $2^{d(X)}$. By our set-theoretic assumption, $2^{d(X)}=\lambda^{+}$for some $\lambda$. Thus there exists a closed discrete $F \subset X$ with $|F|=\lambda^{+}=2^{d(X)}$. By Theorem 7, $X$ does not have property (a).

Proof of (ii). We assume " $\mathfrak{p}=\mathfrak{c}$ and $\mathfrak{c}$ is weakly inaccessible", and construct a counterexample to the statement $\mathcal{S}$. Our counterexample will be of the form $\Psi(\mathcal{A}) \cup\{\infty\}$, where $\mathcal{A}$ is a special kind of almost disjoint family, and " $\infty$ " is one additional point.

Let $\mathcal{T}$ be a tower on $\omega$. By "p $=\mathfrak{c}$ ", we may assume without loss of generality that $\mathcal{T}=\left\{T_{\alpha}: \alpha<\mathfrak{c}\right\}$, and for all $\alpha<\beta<\mathfrak{c}, T_{\beta} \backslash T_{\alpha}$ is finite, and $A_{\alpha}=T_{\alpha} \backslash T_{\alpha+1}$ is infinite. Clearly, $\mathcal{A}=\left\{A_{\alpha}: \alpha<\mathfrak{c}\right\}$ is an almost disjoint family. Let $x_{\alpha}$ be the point of $\Psi(\mathcal{A})$ associated with $A_{\alpha}$. Put $X=\Psi(\mathcal{A}) \cup\{\infty\}$, let points of $\Psi(\mathcal{A})$ have their usual neighborhoods, and define basic neighborhoods of " $\infty$ " for each $\alpha<\mathfrak{c}$ and finite $H \subset \omega$ by

$$
W(\alpha, H)=\{\infty\} \cup\left(T_{\alpha} \backslash H\right) \cup\left\{x_{\beta}: \alpha \leq \beta<c\right\} .
$$

It is straightforward to show that $X$ is a zero-dimensional $T_{2}$-space.

Since $W(\alpha, \emptyset)$ misses the closed discrete set $\left\{x_{\beta}: \beta<\alpha\right\}$, and $\mathfrak{c}$ is a limit cardinal, it is clear that $e(X) \geq \mathfrak{c}$, and since $|X|=\mathfrak{c}$, in fact $e(X)=\mathfrak{c}$ (however, $X$ has no closed discrete set of cardinality $\mathfrak{c}$ since " $\infty$ " is a limit point of any set of cardinality $\mathfrak{c})$. Since $X$ is separable, $d(X)=\omega$, and thus we have $e(X)=\mathfrak{c}=2^{d(X)}$. It remains to show that $X$ satisfies property (a). Let $\mathcal{U}$ be an open cover of $X$, and $D$ a dense set. There exists $\alpha<\mathfrak{c}, U_{\alpha} \in \mathcal{U}$, and finite $H_{\alpha} \subset \omega$ such that $W\left(\alpha, H_{\alpha}\right) \subset U_{\alpha}$. Pick $d_{0} \in T_{\alpha} \backslash H_{\alpha} \subset U_{\alpha}$. For $\beta<\alpha$, there exist finite sets $F_{\beta}$ and $U_{\beta} \in \mathcal{U}$ such that

$$
\left\{x_{\beta}\right\} \cup\left(A_{\beta} \backslash F_{\beta}\right) \subset U_{\beta} \text { and } A_{\beta} \backslash F_{\beta} \subset \omega \backslash T_{\alpha} .
$$


Now $\mathcal{A}^{\prime}=\left\{A_{\beta} \backslash F_{\beta}: \beta<\alpha\right\}$ is an almost disjoint family with $\left|\mathcal{A}^{\prime}\right|<\mathfrak{c}=$ $\mathfrak{p}$; so by Theorem $3, \Psi\left(\mathcal{A}^{\prime}\right)$ satisfies property (a). Thus there exists a closed discrete $P \subset \omega$ such that $\operatorname{st}(P, \mathcal{U})=\Psi\left(\mathcal{A}^{\prime}\right)$. Finally, we note that

$$
Z=\omega \backslash\left(\operatorname{st}(P, \mathcal{U}) \cup\left(T_{\alpha} \backslash H_{\alpha}\right)\right)
$$

is a closed discrete subset of $X$. Thus $P \cup Z \cup\left\{d_{0}\right\}$ is closed discrete in $X$, $P \cup Z \cup\left\{d_{0}\right\} \subset \omega \subset D$, and

$$
\operatorname{st}\left(P \cup Z \cup\left\{d_{0}\right\}, \mathcal{U}\right)=X .
$$

Thus $X$ satisfies property (a), and this completes the proof.

REMARK 1. In the proof of Theorem 9(ii), we used the statement " $c$ is a limit cardinal." In the presence of " $\mathfrak{p}=\mathfrak{c}$ ", this statement is equivalent to "c is weakly inaccessible" because $\mathfrak{p}$ is regular (see $[2,3.1]$ or $[5,21 \mathrm{E}]$ ).

7. Using only dense sets of smallest cardinality. It seems natural to ask if we can find the numbers $d d(X)$ and $d d c(X)$ by looking only at dense sets of cardinality $d(X)$. To consider this, we define

$$
d d_{1}(X)=\min \left\{\left|\mathcal{F}_{D}\right|: D \text { is dense and }|D|=d(X)\right\},
$$

and

$$
d d c_{1}(X)=\min \left\{|\mathcal{C}|: D \text { is dense and }|D|=d(X) \text { and } \mathcal{C} \text { is cofinal in } \mathcal{F}_{D}\right\} .
$$

We ask whether $d d(X)=d d_{1}(X)$, and $d d c(X)=d d c_{1}(X)$.

Theorem 10. (1) If GCH holds, then for every $T_{1}$-space $X, d d(X)=$ $d d_{1}(X)$.

(2) If $\mathfrak{p}>\omega_{1}$ then there exists a $T_{3.5}$-space $X$ such that

(i) $d d(X)<d d_{1}(X)$, and

(ii) $d d c(X)<d d c_{1}(X)$.

Proof of (1). By $T_{1}$,

$$
d(X) \leq d d(X) \leq d d_{1}(X) \leq 2^{d(X)}=d(X)^{+} .
$$

Thus we need only consider the case $d(X)=d d(X)$. In this case, there exists a dense set $D$ such that $\left|\mathcal{F}_{D}\right|=d(X)$. By $T_{1},|D| \leq\left|\mathcal{F}_{D}\right|$; so $|D|=d(X)$.

Proof of (2)(i). The example is a subspace of $2^{\omega_{1}}$ with the product topology, and we use well known properties of this space. Let $C \subset 2^{\omega_{1}}$ be a countable dense set. Let $D=\left\{f \in 2^{\omega_{1}}:\left|\left\{\alpha<\omega_{1}: f(\alpha) \neq 0\right\}\right| \leq \omega\right\}$. Put $X=C \cup D$ as a subspace of the space $2^{\omega_{1}}$. It is well known that $D$ is countably compact. Let $E$ be a countable dense subset of $X$. Then $E \cap D \subset D_{\alpha}$ for some $\alpha<\omega_{1}$ where

$$
D_{\alpha}=\left\{f \in 2^{\omega_{1}}: f(\beta)=0 \text { for all } \alpha \leq \beta<\omega_{1}\right\} .
$$


Since $D_{\alpha}$ is a closed nowhere dense set in $2^{\omega_{1}}, E \cap\left(C \backslash D_{\alpha}\right)$ is dense in $C$, therefore dense in $2^{\omega_{1}}$. Pick any $y \in 2^{\omega_{1}} \backslash X$. Since $\chi\left(y, 2^{\omega_{1}}\right)=\omega_{1}$, and $\mathfrak{p}>\omega_{1}$, it is well known that there exists a countably infinite set $H \subset E$ such that $H$ converges to $y$. It follows that $H$ has no limit points in $X$ (since $y \notin X)$; so $H$ is an infinite closed discrete subset of $E$. Thus $\left|\mathcal{F}_{E}\right|=2^{\omega}$, and since $E$ was an arbitrary countable dense subset of $X$, we have $d d_{1}(X)=2^{\omega}$. On the other hand, $D_{0}=\left\{f \in 2^{\omega_{1}}:\left|\left\{\alpha<\omega_{1}: f(\alpha)=1\right\}\right|<\omega\right\}$ is dense in $2^{\omega_{1}}$, and has no infinite closed discrete set in $X$ since $D_{0} \subset D$ (and $D$ is countably compact). Hence $\mathcal{F}_{D_{0}}=\left[D_{0}\right]^{<\omega} ;$ so $\left|\mathcal{F}_{D_{0}}\right|=\omega_{1}$. Thus $d d(X) \leq \omega_{1}$ (in fact, $d d(X)=\omega_{1}$ ). Thus we have

$$
d d(X)=\omega_{1}<\mathfrak{p} \leq 2^{\omega}=d d_{1}(X) .
$$

Proof of (2)(ii). We first note that by the countable compactness of $D$, we have $d d c(X) \leq \omega_{1}$ (in fact, $\left.d d c(X)=\omega_{1}\right)$. Now we show that $d d c_{1}(X)>\omega_{1}$. Let $E$ be a countable dense subset of $X$, and $\left\{H_{\alpha}: \alpha<\omega_{1}\right\}$ a family of closed discrete (in $X$ ) subsets of $E$. Since $X$ is dense in $2^{\omega_{1}}$, it follows that $\mathrm{cl} H_{\alpha}$, the closure of $H_{\alpha}$ in $2^{\omega_{1}}$, is nowhere dense in $2^{\omega_{1}}$. Since $\mathfrak{p}<\omega_{1}, 2^{\omega_{1}}$ is not the union of $\omega_{1}$ nowhere dense sets $[5,14.2]$; so there exists a point $y \in 2^{\omega_{1}}$ such that $y \notin \bigcup\left\{\operatorname{cl} H_{\alpha}: \alpha<\omega_{1}\right\}$. Again using $\mathfrak{p}>\omega_{1}$, there exists a sequence in $E$ converging to $y$, which gives us a closed discrete (in $X$ ) set that is almost disjoint from (and hence not a subset of) each $H_{\alpha}$.

The space $X=C \cup D$ in Theorem 10(2) does not have property (a). This follows from a result of Matveev [12, Proposition 2].

\section{Questions}

1. Can the consistency of $\neg \mathcal{S}$ be established without the use of large cardinals?

2. By Theorem 3, every almost disjoint family $\mathcal{A}$ with $|\mathcal{A}|<\mathfrak{p}$ is soft. Does $|\mathcal{A}|<\mathfrak{a}$ imply that $\mathcal{A}$ is soft (this question was raised by K. Kunen)? If the answer is "no", then we ask the question for $|\mathcal{A}|<b$ (recall that $\mathfrak{p} \leq \mathfrak{b} \leq \mathfrak{a} \leq \mathfrak{c})$

3. Can the set-theoretic assumptions in Theorem 8 be weakened, or in the case of Theorem 10(2)(ii), be eliminated entirely?

\section{References}

[1] M. G. Bell, On the combinatorial principal P(c), Fund. Math. 114 (1981), 149-157.

[2] E. K. van Douwen, The integers and topology, in: Handbook of Set-Theoretic Topology, K. Kunen and J. E. Vaughan (eds.), North-Holland, 1984, 111-167.

[3] R. Engelking, General Topology, PWN, Warszawa, 1977.

[4] W. G. Fleissner and A. W. Miller, On Q-sets, Proc. Amer. Math. Soc. 78 (1980), 280-284. 
[5] D. H. Fremlin, Consequences of Martin's Axiom, Cambridge Univ. Press, Cambridge, 1984.

[6] L. Gillman and M. Jerison, Rings of Continuous Functions, van Nostrand, Princeton, 1960.

[7] S. H. Hechler, Short complete nested sequences in $\beta \boldsymbol{N} \backslash \boldsymbol{N}$ and small maximal almost-disjoint families, Gen. Topology Appl. 2 (1972), 139-149.

[8] R. E. Hodel, Cardinal Functions I, in: Handbook of Set-Theoretic Topology, K. Kunen and J. E. Vaughan (eds.), North-Holland, 1984, 1-61.

[9] W. Just, M. V. Matveev and P. J. Szeptycki, Some results on property (a), Topology Appl., to appear.

[10] K. Kunen, Set Theory, North-Holland, 1980.

[11] M. V. Matveev, Absolutely countably compact spaces, Topology Appl. 58 (1994), 81-92.

[12] -, On feebly compact spaces with property (a), preprint.

[13] - Some questions on property (a), Questions Answers Gen. Topology 15 (1997), 103-111.

[14] M. E. Rudin, I. Stares and J. E. Vaughan, From countable compactness to absolute countable compactness, Proc. Amer. Math. Soc. 125 (1997), 927-934.

Department of Mathematics

Ohio University

Athens, Ohio 45701

U.S.A.

E-mail: szeptyck@bing.math.ohiou.edu
Department of Mathematical Sciences University of North Carolina at Greensboro Greensboro, North Carolina 27412 U.S.A.

E-mail: vaughanj@steffi.uncg.edu 